\title{
Noncalcified pancreatic stone treated with electro- hydraulic lithotripsy using SpyGlass pancreatoscopy
}

Endoscopic removal of pancreatic stones remains challenging, especially when the stones are larger than $1 \mathrm{~cm}$ in diameter. Although electrohydraulic lithotripsy (EHL) with a baby endoscope is useful in some cases [1], it can be difficult because of poor operability of the baby scope or incomplete irrigation with the EHL probe loaded in the working channel of the scope. Recently, the SpyGlass Direct Visualization System (SDVS; Boston Scientific, Natick, Massachusetts, USA) has allowed more effective diagnostic and therapeutic applications [2,3]. Here we report the successful fragmentation of a large noncalcified pancreatic stone using SDVS-guided EHL.

A 58-year-old man with alcoholic chronic pancreatitis was referred to our hospital for management of recurrent pancreatitis. Computed tomography (CT) showed a noncalcified stone of $1.5 \mathrm{~cm}$ in diameter in the dilated main pancreatic duct (৫ Fig. 1).

Because of the difficulty of targeting noncalcified stones under fluoroscopic guidance for extracorporeal shockwave lithotripsy (ESWL), we employed EHL under the guidance of SpyGlass pancreatoscopy instead.

Following pancreatic sphincterotomy and balloon dilation of a stricture within the main pancreatic duct in the head of the pancreas, a large white stone was visualized by SDVS although it was located just behind a bend within the duct ( $\bullet$ Fig. 2). EHL was applied using a lithotriptor (Northgate Autolith; Nortech, Elgin, Illinois, USA) and the stone was disintegrated under direct visualization combined with frequent irrigation through the dedicated channel $($ Video 1$)$.

Complete clearance of the main pancreatic duct was obtained and the patient was

\section{Video 1}

Electrohydraulic lithotripsy (EHL) performed to disintegrate a large pancreatic stone using the SpyGlass Direct Visualization System.

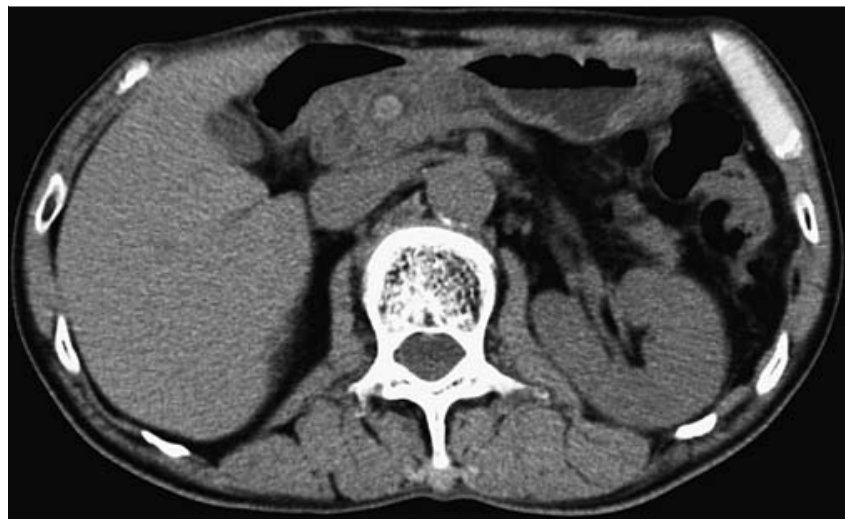

Fig. 1 Computed tomography (CT) scan showing a noncalcified stone of $1.5 \mathrm{~cm}$ in diameter in the dilated main pancreatic duct.

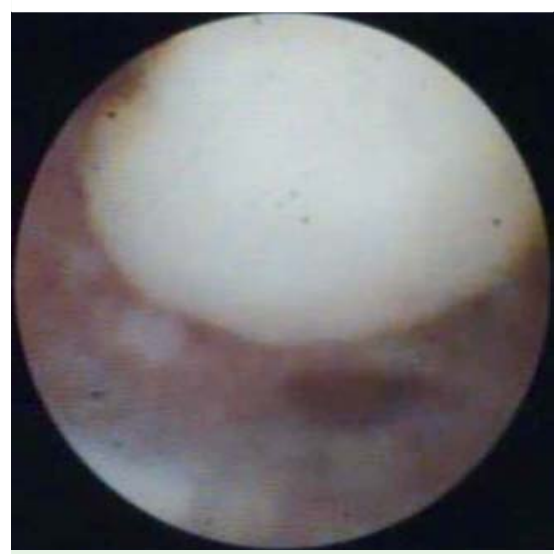

Fig. 2 View through the SpyGlass Direct Visualization System showing a large white stone in the main pancreatic duct.

discharged with complete resolution of his pain.

EHL for pancreatic stones guided by a conventional baby endoscope is very difficult because of poor visualization in the tortuous and narrow pancreatic duct and inefficient irrigation. As the SDVS has 4-way tip deflection and a dedicated irrigation channel, we were able to successfully perform fragmentation of the pancreatic stones by SDVS-guided EHL. The efficacy of SDVS in the management of pancreatic stones needs to be assessed in further cases.

Endoscopy_UCTN_Code_TTT_1AR_2AH

Competing interests: None
N. Sasahira ${ }^{1}$, H. Isayama ${ }^{1}$, R. Nagano ${ }^{1}$, H. Kogure ${ }^{1}$, K. Yamamoto ${ }^{1}$, K. Kawakubo ${ }^{1}$, N. Yamamoto ${ }^{1}$, K. Hirano ${ }^{1}$, M. Tada ${ }^{1}$, K. Kubota ${ }^{2}$, K. Koike ${ }^{1}$ 1 Department of Gastroenterology, Graduate School of Medicine, The University of Tokyo, Japan

2 Department of Gastroenterology, Graduate School of Medicine, Yokohama City University, Japan

References

1 Howell DA, Dy RM, Hanson BL et al. Endoscopic treatment of pancreatic duct stones using a $10 \mathrm{~F}$ pancreatoscope and electrohydraulic lithotripsy. Gastrointest Endosc 1999; 50: 829-833

2 Chen YK. Preclinical characterization of the Spyglass peroral cholangiopancreatoscopy for direct access, visualization, and biopsy. Gastrointest Endosc 2007; 65: 303 -311

3 Baron TH, Saleem A. Intraductal electrohydraulic lithotripsy by using SpyGlass cholangioscopy through a colonoscope in a patient with Roux-en-Y hepaticojejunostomy. Gastrointest Endosc 2010; 71: 650-651

Bibliography

DOI $10.1055 / \mathrm{s}-0030-1256600$

Endoscopy 2011; 43: E272

(c) Georg Thieme Verlag KG Stuttgart · New York . ISSN 0013-726X

Corresponding author

H. Isayama, MD, PhD

Department of Gastroenterology

Graduate School of Medicine

The University of Tokyo

7-3-1 Hongo, Bunkyo-ku

Tokyo 113-8655

Japan

Fax: +81-33-8140021

isayama-tky@umin.ac.jp 